\title{
ANÁLISIS DE UN EDIFICIO HISTÓRICO USANDO MEF: EL PUENTE LA LIBERTAD, EN BUGA - VALLE DEL CAUCA (1874)
}

\author{
ANALYSIS OF AN HISTORICAL BUILDING USING MEF: \\ THE BRIDGE LA LIBERTAD, IN BUGA - THE VALLEY OF THE CAUCA (1874)
}

\author{
Jorge Alberto, Galindo Díaz \\ Arquitecto, Ph.D., Prof. Asociado - Escuela de Arquitectura y Urbanismo, \\ Universidad Nacional de Colombia, sede Manizales, jagalindod@unal.edu.co \\ Jairo Andrés, Paredes López \\ Ingeniero Civil, Ms.C., Prof. Asociado - Departamento de Ingeniería Civil, \\ Universidad Nacional de Colombia, sede Manizales, japaredesl@unal.edu.co \\ Diego Fernando, Mora Méndez \\ Ingeniero Civil, Auxiliar de investigación, \\ Universidad Nacional de Colombia, sede Manizales, dfmoram@unal.edu.co
}

Fecha de recepción: Junio 26 de 2007

Fecha de aprobación: Septiembre 7 de 2007

\section{RESUMEN}

Este documento presenta parte de los resultados de un proyecto de investigación mucho más amplio que ha reconocido aspectos históricos y técnicos propios de un conjunto de 34 puentes históricos de arco de ladrillo, de los cuales aun existen 20, todos con características geométricas diversas y dimensiones singulares. Se expone el proceso de modelamiento numérico mediante el método de elementos finitos (MEF) adelantado en uno de ellos y se presentan conclusiones que se pueden extender a la casi totalidad de los casos estudiados con el fin de ofrecer nuevas alternativas de análisis a los profesionales interesados en la rehabilitación y conservación del patrimonio arquitectónico e ingenieril colombiano.

PALABRAS CLAVE: puentes de arco de ladrillo, análisis estructural, método de elementos finitos.

\begin{abstract}
This document presents partial results of a much broader research project that has recognized historical and technical aspects pertaining to a set of 34 historic brick arch bridges, 20 of which still exist, all with diverse geometric characteristics and singular dimensions. The methodological process is exposed as carried out with one of them and conclusions are presented, which can be extended to almost the totality of the cases studied for the purpose of offering new analysis alternatives to professionals interested in the rehabilitation and conservation of the Colombian architectural and engineering patrimony.
\end{abstract}

KEY WORDS: Masonry arch bridges, Structural analysis, Finite element modeling. 


\section{INTRODUCCIÓN}

El desconocimiento en torno al real comportamiento mecánico y la capacidad resistente de puentes antiguos de arco de ladrillo ha sido uno de los más importantes factores para que la ingeniería moderna no los considere como estructuras útiles a la infraestructura vial de algunas naciones. Mientras que en Inglaterra, por ejemplo, existen actualmente más de 60 mil puentes de esta naturaleza en servicio para ferrovías y autovías, en Colombia su uso es concebido como un mal necesario o cuando menos temporal: solo el $1 \%$ de los puentes vinculados a su red vial nacional son de ladrillo [12].

Con el fin de analizar uno de los puentes de ladrillo que en Colombia todavía se encuentra en servicio vehicular, se propuso emplear el Método de Elementos Finitos (MEF) a través de un programa informático comercial el cual permitiría obtener una información aproximada del real comportamiento estructural del puente. Su aplicación en el estudio de este tipo de edificios históricos representa una herramienta muy útil para el objetivo propuesto, especialmente si de lo que se trata es de asumir ese estudio para su conservación y rehabilitación [7]. Como parte de las tareas previas, fue necesario llevar a cabo un estudio histórico capaz de dar cuenta del origen del puente y una caracterización de sus materiales constitutivos.

\section{CONTEXTO GENERAL}

La ciudad de Buga está situada a $3^{\circ} 54^{\prime} 07^{\prime \prime}$ de latitud norte, ligeramente recostada sobre las estribaciones de la Cordillera Central colombiana y en la zona en donde se hace más angosto el valle geográfico del río Cauca. Su área urbana central se ubica en la margen derecha del río Guadalajara que nace de la confluencia de varias quebradas situadas entre los 1.850 y los 3.700 msnm, generando en ocasiones flujos de caudal máximo instantáneo.
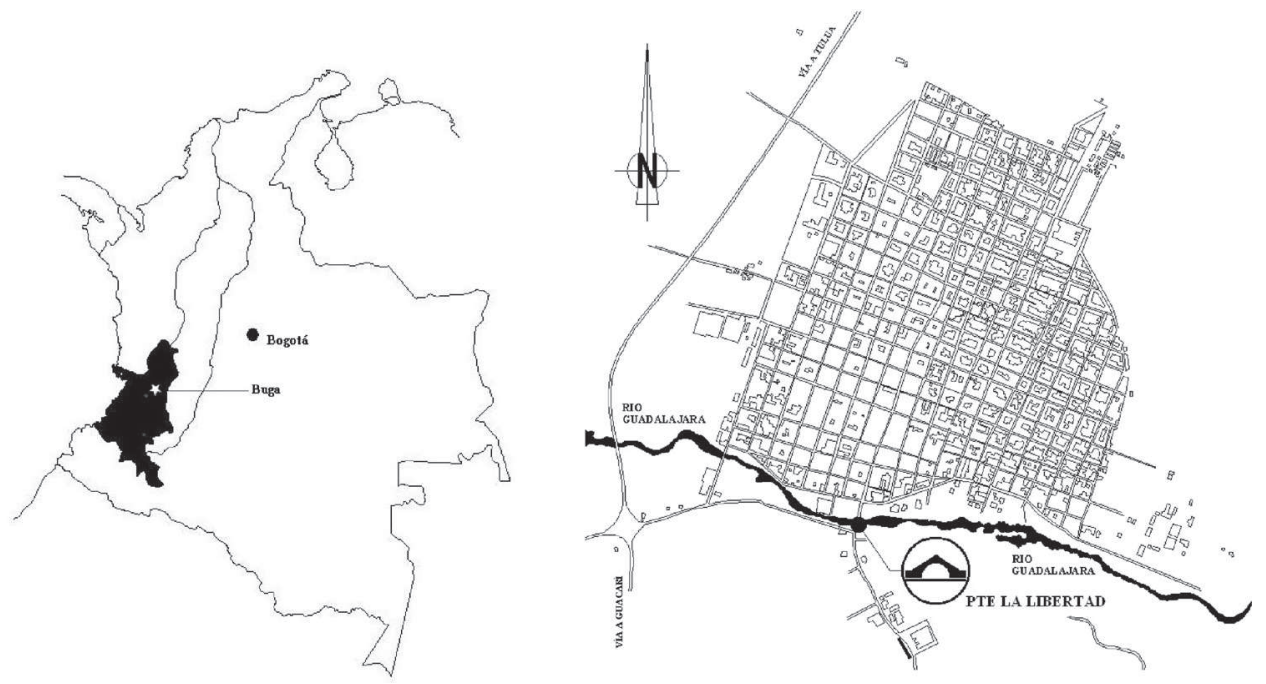

Figuras 1A y 1B. Localización del área de investigación.

Izquierda: Localización sobre el territorio colombiano. Derecha: Localización urbana. 
El puente de La Libertad, sobre el río Guadalajara, hace parte del camino que desde el período colonial vinculaba a Cali y Popayán con otras poblaciones del sur - occidente de Colombia, paralelo al sentido N-S del río Cauca, e importante para la circulación de productos agrícolas y mineros que hicieron de la región una de las más ricas del país.

\subsection{HISTORIA DE LA CONSTRUCCIÓN DEL PUENTE LA LIBERTAD}

Los trabajos de construcción del puente se iniciaron en 1874 gracias a un contrato firmado entre el señor Antonio María Soto -presidente de la Junta constituida para tal fin- y el ingeniero colombiano Modesto Garcés. Mediante este documento, el segundo se obligaba a construir un puente sobre el río Buga, conforme a los diseños que poseía la Junta y según los cuales, este debía hacerse mediante tres arcos levantados sobre estribos de piedra labrada por el exterior, desde flor de tierra hasta el arranque de los arcos. Es interesante señalar también que en el contrato se estipulaba que las bóvedas debían sujetarse a las reglas que la ciencia aconseja, soportando su propio peso, el de los terraplenes, calzada y demás accesorios y soportando una sobre carga de 12.500 kilogramos.

Desde sus comienzos, la estructura se consideraba como una entidad formada por tres partes claramente diferenciadas: (a) el tramo central del puente de 3 arcos sobre el río, (b) los terraplenes, y (c) el viaducto formado también por 5 arcos, todos de mampostería de ladrillo. En un informe de 1875, el propio Garcés lo expresaba de la siguiente manera:

El puente de Buga (...) es una obra mixta que comprende tres partes principales: el puente propiamente dicho que abarca con tres grandes arcos de a 10 metros de luz cada uno y el grosor de sus pilares y estribos un espacio de 41 metros; la segunda parte consta de los terraplenes indispensables para el descenso de la altura del puente en un espacio de 50 metros; y la tercera parte la forma un viaducto de cinco arcos espaciosos á distancias proporcionadas, adecuándolos á los accidentes del terreno en un espacio de 50 metros. Tomado de Registro Oficial del Cauca, No. 94, abril 17 de 1875.

Este dato histórico es particularmente importante para el proceso de análisis mecánico del macro - modelo que se habría de elaborar ya que permitió considerar el tramo central del puente como una estructura independiente vinculada a las demás partes mediante un par de macizos romboidales que bien podían considerarse como resultados de la unión de las aletas del tramo central con las dos vías de acceso, una a cada lado. Haciendo uso de procesos de digitalización en tres dimensiones se reconstruyeron dos momentos en el proceso constructivo, tal como se muestran en las Figuras 2A y $2 \mathrm{~B}$.

El plazo fijado para la terminación de los trabajos era de 4 años, período durante el cual el propio Garcés quedaba con el carácter de recaudador de las contribuciones impuestas en el Municipio para las obras del puente y canalización del río. Adicionalmente, el ingeniero recibía en pago tanto la mano de obra de los presidiarios, algunos materiales disponibles (ladrillos y maderas principalmente), el producto de la contribución sobre mortuorias, las 
donaciones voluntarias, las limosnas que la iglesia recogiera para tal fin y 4 mil pesos que la Legislatura del Estado Soberano del Cauca había aprobado en calidad de auxilio; todo ello hasta sumar los $\$ 28.000$ que costaría el puente. En el ya citado informe de 1875 se describía de la siguiente manera el avance de las obras a pocos meses de su inicio:

Hay un arco de 10 metros de luz terminado; otro á la altura de los riñones, con las cimbras arregladas para continuar cerrándolo. Para cerrar el tercer arco, que también está adelantado, y por supuesto terminados los estribos y muros laterales, tendré necesidad de descimbrar el primero (cuando el $2^{\circ}$ esté terminado) y construirlo sobre estas cimbras ... Omito todos los detalles de construcción y cálculo de volumen, presión \& por considerarlos innecesarios y no adecuados a la naturaleza de este informe (...) su valor excederá en mucho a la suma del contrato.

Es decir que se reutilizaban las potentes cimbras sobre las que habrían de descansar más de los 38 mil ladrillos con los que se decía contar, fabricados en un galpón del que se disponía con exclusividad y los que se pegaban usando las cales traídas desde la cercana población de Vijes. Posteriormente, en fecha incierta, pero antes de ser puesto en servicio a comienzos del siglo XX, se construyeron los viaductos de nivelación: el del lado norte consta de 9 arcos de medio punto de luz variable, y el del lado sur de solo 3 arcos, también de luz variable, todos en ladrillo.
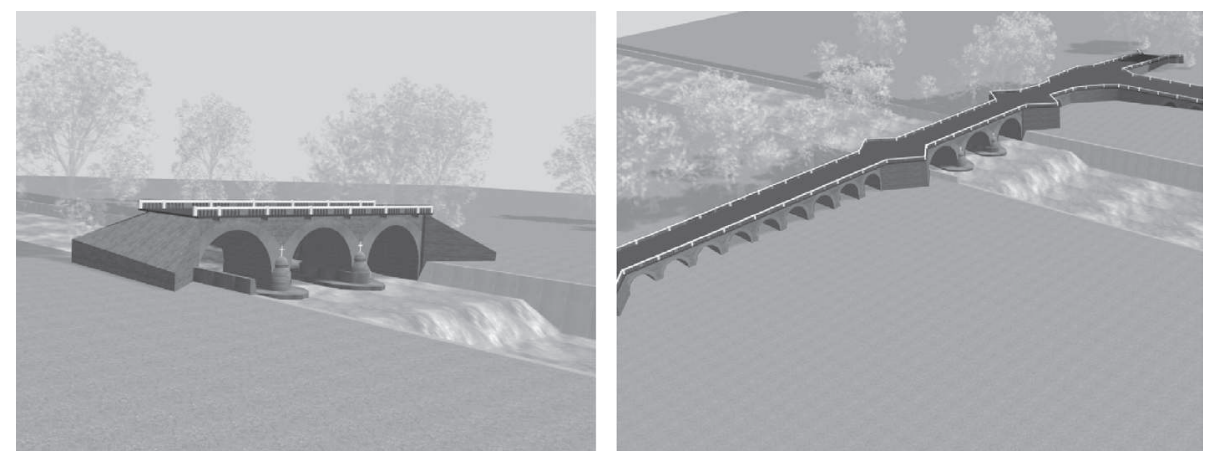

Figuras 2A y 2B. Reconstrucción de dos momentos del proceso constructivo. Izquierda: primera fase - construcción del tramo central.

Derecha: segunda fase - construcción de terraplenes y viaductos

\subsection{CARACTERÍSTICAS DIMENSIONALES Y ESTADO DE CONSERVACIÓN}

Un levantamiento arquitectónico realizado en septiembre de 2005 verificó las dimensiones a las que hacían referencia los documentos históricos. Además, se observó que el ancho del puente era de 6,00 m constantes, aunque una intervención efectuada a mediados del siglo $\mathrm{XX}$ mediante vigas de hormigón en voladizo a cada lado, aumentó su ancho total a los $8,00 \mathrm{~m}$. 


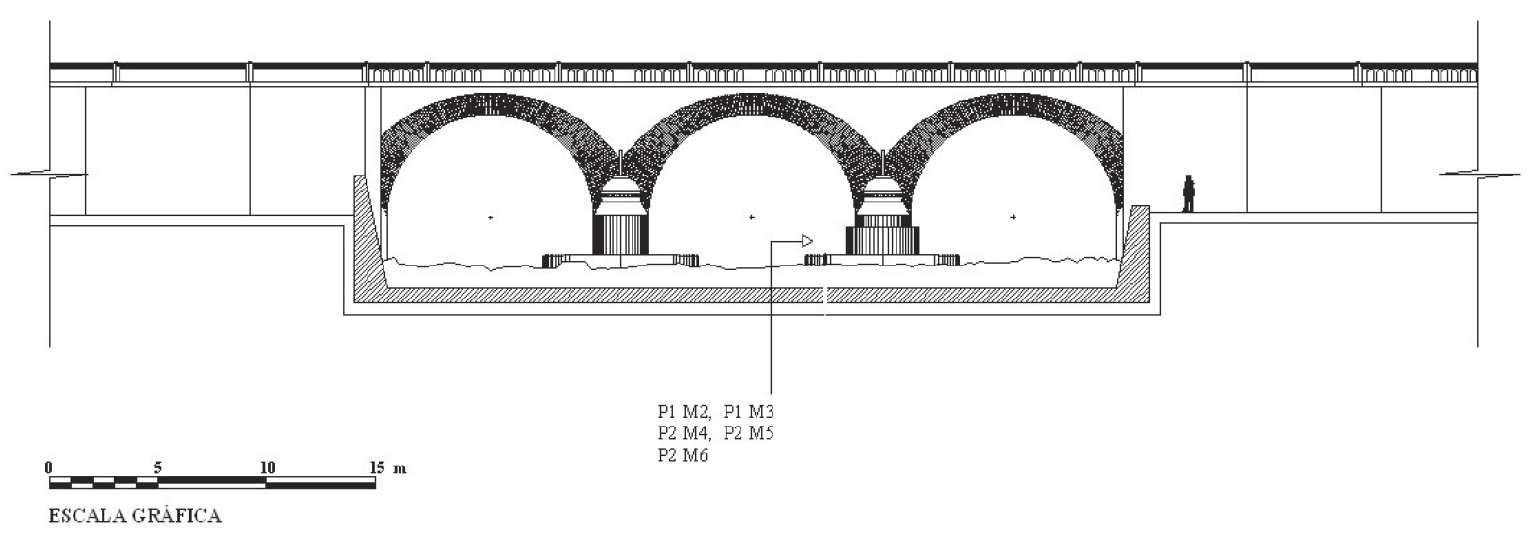

Figura 3. Alzada del tramo central del puente.

En el tramo central todos los arcos son de medio punto con un diámetro de 9,51 $\mathrm{m}$, en tanto que la línea de sus impostas se encuentra siempre al mismo nivel. Los ladrillos de las roscas acusan una disposición radial con trama idéntica y dimensiones iguales: 0,95 $\mathrm{m}$ en la clave y 1,82 m en los riñones. La altura libre entre el intradós de la clave y el nivel medio de las aguas es de 7,00 m. El ancho de las pilas centrales es de 2,53 m. En su tramo más alto, los tímpanos alcanzan una altura de $4,09 \mathrm{~m}$ y su espesor equivale a un muro en doble tizón, es decir, $80 \mathrm{~cm}$.

El puente se encuentra en buen estado de conservación aunque las bases de las pilas del tramo central presentan un acusado deterioro a causa de la acción erosiva de las aguas del río Guadalajara obligando a un recalce en hormigón armado de tipo superficial construido recientemente (c.a. 1990). La inspección de 2005 determinó además que existen cavidades bajo las pilas por socavamiento de la acción hídrica.

\section{MATERIALES Y MÉTODOS}

A partir de la investigación bibliográfica, la documentación histórica y la inspección visual se asumió que el puente estaba constituido por dos materiales: (a) la mampostería y (b) el material de relleno de las cavidades existentes entre las bóvedas, los tímpanos y el tablero. El material de rodamiento y el hormigón de las adiciones posteriores se consideran como sobrepeso.

En el caso del puente La Libertad, la mampostería es un material compuesto por ladrillos de dimensiones más o menos fijas $(20 \times 40 \times 10 \mathrm{~cm})$ fabricados a pie de obra y organizados conforme a una determinada disposición geométrica, unidos entre sí por el mortero de pega, el cual a su vez está elaborado por una mezcla de cal y arena en proporción 1:3, conforme a lo pactado en el contrato original de 1875. Por su parte, el material de relleno sobre las bóvedas del puente está conformado por una mezcla de tierra negra, piedra de canto rodado y fragmentos de ladrillo. Se sabe hoy que el relleno juega un papel importante 
en tanto que afecta significativamente el comportamiento del conjunto puesto que: (a) su peso propio colabora en la estabilidad estructural y (b) su masa contribuye a distribuir las cargas concentradas sobre la superficie de rodamiento [13], [5].

Con el fin de alimentar el programa informático de elementos finitos se hizo necesario definir el comportamiento elástico de los todos estos materiales cuantificando de la manera más precisa posible al menos 3 valores: (a) el coeficiente de Poisson, (b) el módulo de elasticidad y (c) el peso unitario. La ausencia de información característica de materiales propios del tiempo y lugar del puente estudiado hizo entonces necesario aplicar diversas pruebas en el Laboratorio de Resistencia de Materiales de la Universidad Nacional de Colombia, sede Manizales.

\subsection{CARACTERIZACIÓN DE LA MAMPOSTERÍA}

Para obtener muestras de ladrillo y mortero, ubicados en la zona de los arcos y los tímpanos, se hizo extracción de núcleos haciendo uso de un taladro extractor tipo HILTI modelo DDBCQ-S. Luego de fijar el taladro al plano del arco o el tímpano, se realizó la extracción del núcleo utilizando en ello una broca de $0,4 \mathrm{~m}$ de longitud y $0,051 \mathrm{~m}$ de diámetro interior.

A cada una de las muestras de ladrillo se aplicó un conjunto de pruebas de laboratorio siguiendo las recomendaciones de la norma NTC 682 [9] mediante la cual se determina el módulo de resistencia a la compresión en frío. A través de ellas se logró la obtención de los datos recogidos en la Tabla 1.

Tabla 1. Datos correspondientes a las muestras de ladrillo obtenidas del puente La Libertad

\begin{tabular}{|c|c|c|c|c|}
\hline Muestra & $\begin{array}{c}\text { Gravedad } \\
\text { específica } \\
\text { aparente } \mathbf{( T )}\end{array}$ & $\begin{array}{c}\text { Densidad } \\
\text { Aparente } \\
\left(\mathbf{g r} / \mathbf{c m}^{3} \text { ) }\right.\end{array}$ & $\begin{array}{c}\text { Resistencia a } \\
\text { la Compresión } \\
\text { en Frío (MPa) }\end{array}$ & $\begin{array}{c}\text { Módulo de } \\
\text { Elasticidad (MPa) }\end{array}$ \\
\hline P1 M2-1 & 2,76 & 1,38 & 8,27 & 339,53 \\
\hline P1 M2-2 & 2,73 & 1,69 & 7,58 & 309,16 \\
\hline P1 M3-1 & 2,87 & 1,59 & 7,27 & 347,08 \\
\hline P1 M3-2 & 2,71 & 1,73 & 5,24 & 303,97 \\
\hline P2 M4-1 & 2,8 & 1,7 & 8,19 & 339,05 \\
\hline P2 M4-2 & 2,76 & 1,72 & 7,94 & 319,89 \\
\hline P2 M5 & 2,65 & 1,78 & 8,16 & 297,54 \\
\hline P2 M6 & 2,4 & 1,83 & 6,20 & 183,36 \\
\hline Valor promedio simple & 2,71 & 1,67 & 7,35 & 304,94 \\
\hline
\end{tabular}

En las muestras obtenidas en la zona inferior del puente, el espesor de la junta de mortero alcanzó $3,00 \mathrm{~cm}$ de espesor, lo que permitió contar con especímenes en los cuales fue posible aplicar las pruebas mecánicas recomendadas en la norma NTC 220 [10] mediante la cual se determina la resistencia de morteros de cemento hidráulico usando cubos de 50 
$\mathrm{mm}$ de lado. Vale la pena aclarar que en las áreas más altas del puente, el espesor de la junta de mortero se reduce notablemente llegando a alcanzar escasos 0,9 cm. Los datos obtenidos en las pruebas aplicadas a los morteros se recogen en la Tabla 2.

Tabla 2. Datos correspondientes a las muestras de mortero obtenidas del puente La Libertad

\begin{tabular}{|c|c|c|c|c|}
\hline $\begin{array}{c}\text { Muestra } \\
\text { Dimensiones de la base } \\
\mathbf{x} \text { altura } \mathbf{( c m})\end{array}$ & $\begin{array}{c}\text { Peso de la } \\
\text { muestra } \\
\mathbf{( g r})\end{array}$ & $\begin{array}{c}\text { Peso } \\
\text { Unitario } \\
\left(\mathbf{g r} / \mathbf{c m}^{3}\right)\end{array}$ & $\begin{array}{c}\text { Resistencia a la } \\
\text { Compresión en } \\
\text { Frío (MPa) }\end{array}$ & $\begin{array}{c}\text { Módulo de } \\
\text { Elasticidad } \\
\mathbf{( M P a )}\end{array}$ \\
\hline $\mathrm{M} 1(3,65 \times 3,62 \times 2,64)$ & 48,93 & 1,397 & 0,57 & 14,16 \\
\hline M2 $(3,13 \times 2,89 \times 2,79)$ & 33,7 & 1,343 & 0,26 & 33,34 \\
\hline M3 $(3,13 \times 2,89 \times 2,79)$ & 56,6 & 1,277 & 0,21 & 6,19 \\
\hline Valor promedio simple & 46,41 & 1,339 & 0,346 & 17,89 \\
\hline
\end{tabular}

Por norma general se asume que en la mampostería, ladrillos y morteros exhiben muy distintas propiedades, dificultando la identificación de una ley única capaz de proveer una descripción global del comportamiento mecánico del conjunto, lo que a su vez hace necesario apelar a los principios de la teoría de la homogenización de la mampostería expuesta por varios autores [1], [3] y [4]. En esta investigación, a partir de los resultados experimentales y de acuerdo con las Ecuaciones (1) y (2), correspondiente a las Ec. D.3.1 y D.3.2, respectivamente, contenidas en la NSR-98, Cap. D.3.7.5, fue posible determinar teóricamente el valor de la resistencia a la compresión de la mampostería del puente:

$$
\begin{gathered}
R_{m}=\left(\frac{2 h}{75+3 h}\right) * f_{c u}^{\prime_{c u}}+\left(\frac{50 k_{p}}{75+3 h}\right) * f_{c p}^{\prime} \leq 0.8 f_{c m}^{\prime_{c m}} \\
f_{m}^{\prime}=0,75 R_{m}
\end{gathered}
$$

Donde $R_{m}$ es el parámetro definido por la Ecuación (1), $h$ es la altura de la unidad de mampostería (en $\mathrm{mm}$ ), $k_{p}$ es un factor de correlación por absorción de la unidad (adimensional) equivalente a 0,8 para unidades de arcilla, $f^{\prime}{ }_{c u}$ es la resistencia a la compresión de las unidades de mampostería (en MPa) y $f^{\prime}{ }_{c p}$ es la resistencia a la compresión del mortero de pega (en $\mathrm{MPa}$ ).

Para determinar el módulo de elasticidad $E_{M}$ de la mampostería se apeló a la Ecuación (3) correspondiente a la Ec. D.5-5 contenida en la NSR-98, Cap. D5.2.1 [2], mediante la cual es posible determinar el módulo de elasticidad para mampostería de arcilla $E_{M}$, tomándolo como función de la resistencia a la compresión de la misma $\left(f^{\prime}{ }_{m}\right)$ :

$$
E_{M}=750 * f_{m}^{\prime} \leq 20.000 M P a
$$


Aunque no se conoce con exactitud el valor del coeficiente de Poisson ( $v$ ) para unidades de mampostería, estudios de carácter experimental han arrojado un rango estimado entre 0,13 y 0,2 dentro del cual éste puede oscilar [11]. Para esta investigación dicho valor se fijó en 0,25 a partir de la Ecuación (4) considerando el valor del módulo de cizallamiento $G$ como equivalente al $40 \%$ del valor del módulo de elasticidad $E$ según la Ec. D.5-7 contenida en la NSR-98, Cap. D5.2.1 [2].

$$
G=\frac{E}{2(1+v)}
$$

El peso unitario de la mampostería se determinó numéricamente sobre un volumen de 1,00 $\mathrm{m}^{3}$, considerando las dimensiones del ladrillo antes señaladas con un espesor promedio de las juntas de mortero valorado en $1,5 \mathrm{~cm}$. Este análisis permitió deducir que el $68,4 \%$ del volumen corresponde a los ladrillos y el 31,6\% restante al mortero de pega y así, mediante un promedio ponderado, obtener un valor para el peso unitario de la mampostería. Un resumen de todos los datos obtenidos para el conjunto de la mampostería se muestra en la Tabla 3.

Tabla 3. Valores asumidos para la mampostería del puente La Libertad

\begin{tabular}{|c|c|c|}
\hline Magnitud & Procedimiento empleado & Valor obtenido \\
\hline Peso unitario & Determinado analíticamente & $1,56 \mathrm{gr} / \mathrm{cm}^{3}$ \\
\hline Resistencia a la compresión $\left(f^{\prime}{ }_{m}\right)$ & Ecuaciones (1) y (2) & $2,97 \mathrm{MPa}$ \\
\hline Módulo de elasticidad $(\mathrm{E})$ & Ecuación (3) & $2225,81 \mathrm{MPa}$ \\
\hline Coeficiente de Poisson & Ecuación (4) & 0,25 \\
\hline
\end{tabular}

El valor de la resistencia a la compresión de la mampostería permitió a su vez establecer los esfuerzos admisibles para compresión axial $\left(F_{a}\right)$ y para compresión por flexión $\left(F_{b}\right)$, haciendo uso de las Ecuaciones (5), (6) y (7) que corresponden a las Ec. D-1-1, D-1-5 y D-1-6 contenidas en la NSR-98, Apéndice D-1 [2].

$$
\begin{gathered}
R_{e}=1-\left(\frac{h^{\prime}}{40 t}\right)^{3} \\
F_{a}=0,2 * f^{\prime}{ }_{m} * R_{e} \\
F_{b}=0,33 * f^{\prime}{ }_{m}
\end{gathered}
$$

De acuerdo con las características dimensionales del puente La Libertad, ya señaladas, la magnitud $h$ ' se asumió como la distancia existente entre el borde superior de los tímpanos y el punto en donde confluyen las bóvedas $(4090 \mathrm{~mm})$ y la magnitud $t$ como el espesor de esos mismos tímpanos (800 mm). Así, el esfuerzo admisible para compresión axial en muros de mampostería no reforzada, para el caso del puente de La Libertad, se fijó en 0,59 MPa y el valor del esfuerzo admisible para compresión por flexión se estimó en 0,98 MPa. 
En cuanto al esfuerzo admisible para tracción que suele presentarse en las juntas de mortero a causa de la flexión que se produce en los muros, se adoptaron los rangos de valores contenidos en el numeral D.1.5.4. de la NSR-98, Apéndice D-1 [2]: entre 0,19 y $025 \mathrm{MPa}$ cuando la tracción se desarrolla de forma perpendicular a la junta horizontal y entre 0,37 y 0,50 MPa cuando es con referencia a la junta vertical.

\subsection{CARACTERIZACIÓN MECÁNICA DEL MATERIAL DE RELLENO}

En términos generales, el material de relleno está compuesto por una matriz de suelo fino combinada con cantos redondeados entre 10 y 15 centímetros de diámetro y trozos de roca muerta (nombre usado en la zona para el suelo residual) que están entre 2 y 3 centímetros de diámetro. Este material térreo cumple varias funciones entre las cuales se cuenta la de servir en la nivelación de la superficie de rodadura o área de tránsito; igualmente proporciona la carga confinante a los arcos para que estos desarrollen su resistencia estructural. En aquellos puentes en los cuales existe tráfico vehicular, el suelo de relleno se encarga de disipar los esfuerzos producidos por las ruedas de los vehículos y transmitir estos esfuerzos disminuidos al arco de ladrillo, elemento estructural que debe soportarlos y transmitirlos a la cimentación del puente.

Con el fin de llevar a cabo la caracterización mecánica se aplicó un conjunto de ensayos de laboratorio sobre muestras alteradas e inalteradas obtenidas en dos apiques. Las muestras alteradas se tomaron para la realización de ensayos de clasificación; las muestras inalteradas se aplicaron para la determinación de los pesos unitarios del suelo y ensayos de resistencia al esfuerzo cortante, específicamente el ensayo de corte directo.

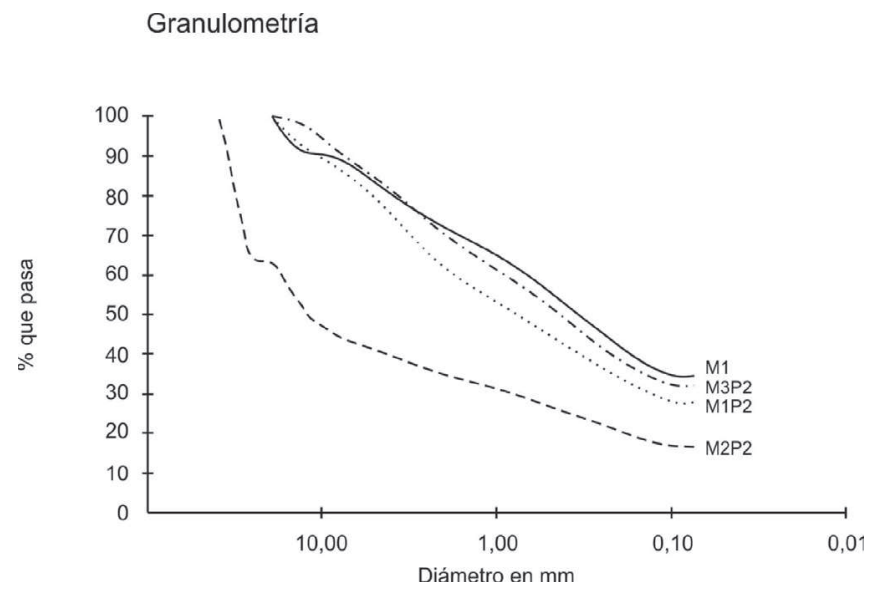

Figura 4. Gráfica de granulometría correspondiente a la muestra obtenida del material de relleno.

Para la clasificación del suelo se hicieron 4 pruebas de granulometría [8] las cuales se relacionan en la Figura 4, en la cual se observan 3 curvas (M1, M1P2 y M3P2) relativamente 
juntas que representan la matriz fina del suelo y la curva M2P2 que representa la granulometría del suelo fino con los fragmentos de roca muerta. Esta diferenciación se hace ya que en el laboratorio fue necesario eliminar los fragmentos de roca muerta de la matriz fina y compactar el suelo de la matriz con el mismo peso unitario de campo; a este suelo se le realizó el ensayo de corte directo, para que así, con los resultados de este ensayo, se pudiesen determinar los parámetros de resistencia al esfuerzo cortante: cohesión y ángulo de fricción interna, al igual que los parámetros elásticos necesarios para la modelación numérica del puente.

Con el fin de fijar los parámetros elásticos a partir de los resultados del ensayo de corte directo y basándose en teorías de mecánica de suelos y de la elasticidad, se determinó el módulo cortante del suelo $G$ a partir de la gráfica esfuerzo cortante $\tau$ versus deformación cortante $\gamma$, utilizando el módulo secante de cada nivel de confinamiento para finalmente establecer un rango de variación de $G$.

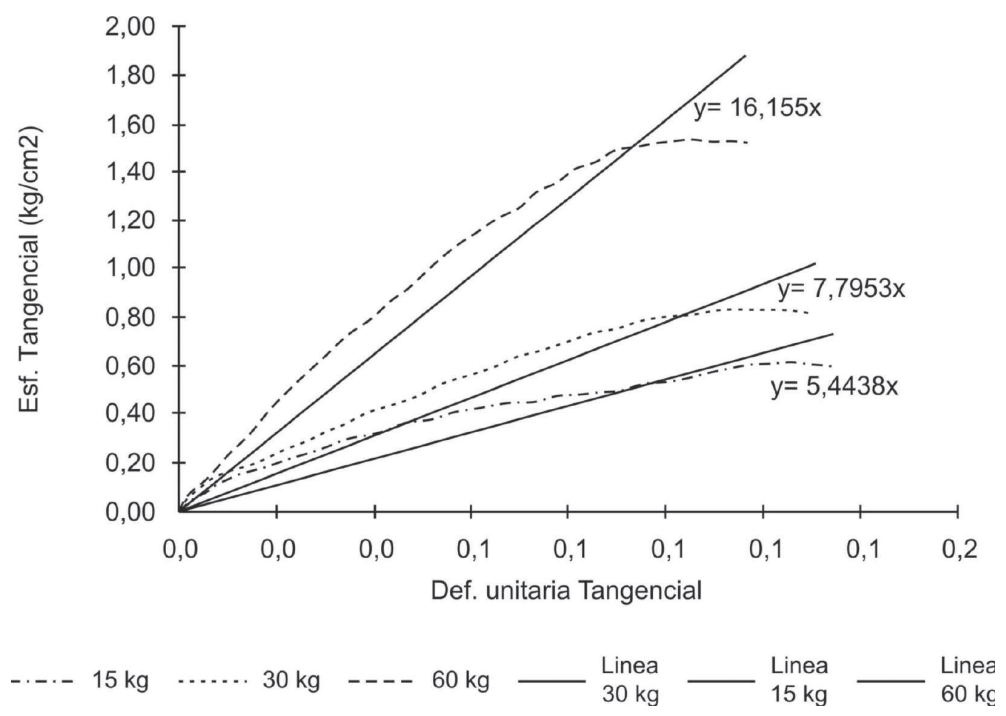

Figura 5. Gráfica de la deformación unitaria tangencial de la muestra del suelo de relleno.

En la Figura 5 la pendiente de cada línea de tendencia representa el valor del módulo para cada nivel de confinamiento del ensayo.

$$
\frac{\sigma_{3}}{\sigma_{1}}=\frac{v}{1-v}
$$

En la relación elástica planteada en la Ecuación (8) se relacionan el esfuerzo principal menor y mayor con la relación de Poisson que se requiere conocer para lo cual, partiendo del principio que el esfuerzo geoestático vertical y el empuje horizontal son esfuerzos principales, se puede plantear la Ecuación (9): 


$$
\frac{\sigma_{3}}{\sigma_{1}}=\frac{\sigma_{h}}{\sigma_{v}}
$$

Conociendo la relación planteada en la Ecuación (10):

$$
\frac{\sigma_{h}}{\sigma_{1}}=k_{o}
$$

Haciendo uso de los principios de la mecánica de suelos se obtuvo el valor de $k_{o}$ a partir de la Ecuación (11),

$$
k_{o}=1-\operatorname{sen} \varphi
$$

donde el ángulo de fricción interna $\varphi$ para el suelo que se tuvo como muestra es de $30^{\circ}$. Así, se determinó que el valor de $k_{o}$ es igual a 0,5 y al igualar las ecuaciones (8), (9) y (10) se obtuvo la siguiente expresión:

$$
\frac{v}{1-v}=k_{o}
$$

De esta manera se determinó que el valor del coeficiente de Poisson ( $v$ ) era igual a 0,33. Nuevamente, se calculó el valor del módulo de elasticidad mediante la Ecuación (4).

Es importante resaltar que en estas relaciones se utilizaron los datos de resistencia al corte propios del suelo para determinar las propiedades elásticas y que los resultados obtenidos en función del nivel de confinamientos se situaron entre 2000 y $8500 \mathrm{kPa}$, valores que comparados con bibliografía de mecánica de suelos [6] están dentro de los valores propuestos para este tipo. El conjunto de los resultados obtenidos para el material de relleno del puente La Libertad se relacionan en la Tabla 4.

Tabla 4. Valores asumidos para el material de relleno del puente La Libertad

\begin{tabular}{|c|c|c|}
\hline Magnitud & Procedimiento empleado & Valor obtenido \\
\hline Valor c de la cohesión $(c)$ & Experimental & $25 \mathrm{KPa}$ \\
\hline Angulo de fricción interno & Experimental & $30^{\circ}$ \\
\hline Módulo de elasticidad $(\mathrm{E})$ & Ecuación (4) & $8500 \mathrm{kPa}$ \\
\hline Angulo de dilatancia & Ecuación (8) & $-0,5$ \\
\hline Coeficiente de Poisson & Ecuación (9) & 0,33 \\
\hline
\end{tabular}

\section{RESULTADOS Y ANÁLISIS}

Para el desarrollo de esta etapa de la investigación se decidió llevar a cabo un análisis del tipo lineal elástico del puente a partir de un macro modelo sometido únicamente a las cargas 
por peso propio. No se consideraron por ahora, efectos producidos por cargas sísmicas, socavaciones o empujes del terreno. Una segunda fase de la investigación, actualmente en curso, se encarga del análisis estructural bajo la acción de cargas horizontales externas.

Como herramienta de análisis se usó el software ANSYS (c) v.10, que cuenta con elemento tipo solid, particularmente solid95 y solid65. En la realización del modelo se siguieron cuatro etapas: (a) reconstitución geométrica a partir de levantamientos elaborados en AutoCAD, (b) enmallado del modelo, (c) asignación de propiedades de los materiales, restricciones y cargas, y (d) solución final del modelo.

\subsection{ANÁLISIS NUMÉRICO PRELIMINAR}

Inicialmente se realizó un modelo simplificado de 3 arcos según la geometría propia del puente asumiendo una serie de consideraciones en las condiciones de frontera. En las caras verticales de los extremos del eje principal del puente se consideró que: (a) los desplazamientos eran nulos en la dirección del eje principal, por confinamiento longitudinal, (b) el desplazamiento en la dirección transversal al eje principal del puente era igual a cero (por efecto de las aletas del puente y los tajamares situados en la base de las pilas), y (c) existían desplazamientos en el sentido vertical libre con el fin de dar continuidad al movimiento del puente en su modelo completo. En los apoyos inferiores se consideraron restringidos todos los desplazamientos y a efectos de simplificar el análisis solo se consideró el valor de la carga muerta.

Para el modelamiento numérico se tuvieron en cuenta tres componentes estructurales que conforman constructivamente esta clase de puentes: las bóvedas, los tímpanos (también llamados pretiles) y el material de relleno, siguiendo pautas de análisis aplicadas en estructuras similares [11]. No se establecieron diferencias para la mampostería de las bóvedas y los tímpanos. Considerando que los programas de elementos finitos no tienen un módulo particular de conversión de unidades, los datos se ingresaron en unidades consistentes del SI.

En el proceso de enmallado y con el fin de lograr continuidad de los elementos, se hicieron coincidir los nodos de todos ellos, sin importar que pertenecieran a diferentes sólidos. Una malla mejor organizada se logró a partir del uso de hexaedros, creando además elementos de longitudes similares en el perímetro de cada área; para este caso se escogió una longitud de $40 / 50 \mathrm{~cm}$.

\subsection{GRÁFICAS DE ESFUERZOS Y ANÁLISIS DE RESULTADOS}

Con el fin de facilitar la lectura y análisis de los resultados y en virtud de la escala de desplazamientos y esfuerzos globales se consideró pertinente descomponer la estructura en sus partes constitutivas ya señaladas usando para ello las herramientas de post - proceso que brinda el software. Esto no significa que se hayan realizado tres modelos distintos e 
independientes; solo para efectos explicativos se muestran por separado los resultados del modelamiento numérico y análisis en las bóvedas y el material de relleno para luego dar paso a un análisis del comportamiento global.

\subsubsection{Análisis del comportamiento estructural de las bóvedas}

El análisis lineal-elástico realizado a partir de la Figura 6 permite apreciar que cada una de las bóvedas del puente es objeto de esfuerzos de compresión que oscilan en un rango de valores entre $29,42 \mathrm{kPa}$ y $172,67 \mathrm{kPa}$. Simultáneamente, las mayores tracciones $(82,65$ $\mathrm{kPa}$ ) se presentan en el sentido longitudinal o eje $X$ sobre la zona baja de los arcos donde los tímpanos tienen mayor rigidez con valores superiores a la mayor tracción en el eje $Y$ que es de $60,5 \mathrm{kPa}$ y al mayor valor obtenido en el eje $Z$ que es de 2,88 kPa. Es importante entonces considerar la vulnerabilidad de estas áreas en caso de sismo o del efecto de cualquier otra fuerza horizontal que afecte su rigidez por fractura.

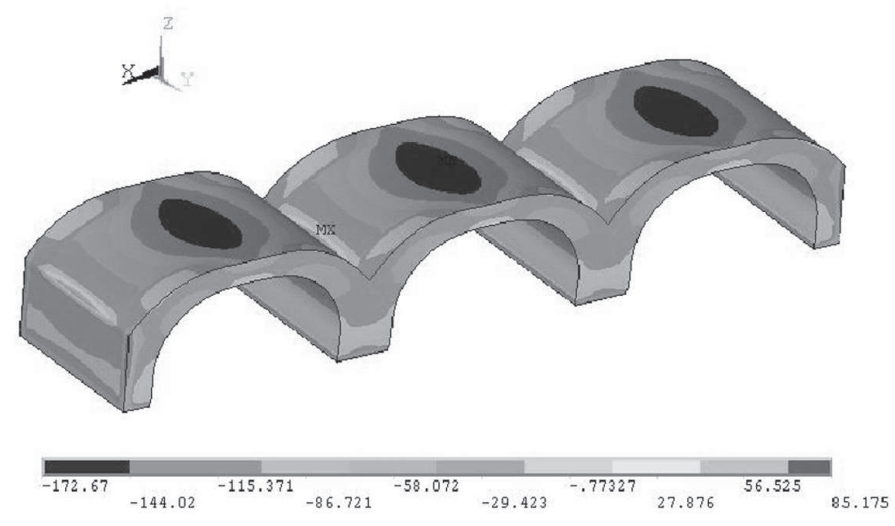

Figura 6. Distribución de esfuerzos en el eje X en la zona de las bóvedas del puente La Libertad.

El análisis de la Figura 7 permite comprender que los mayores esfuerzos a compresión se concentran en las bases de las pilas centrales y tienen un valor aproximado de $500 \mathrm{kPa}$. Por su parte, las roscas de las bóvedas están sometidas en su mayoría a esfuerzos de compresión: la gama de tonos indica valores que oscilan entre $108,9 \mathrm{kPa}$ y $444,21 \mathrm{kPa}$. Se observa también que hay una presencia notable de esfuerzos de tracción sobre la parte superior de cada rosca como lo indica el tono más oscuro. No obstante, es de resaltar que esta condición se debe a la interacción con el material de relleno el cual se encuentra dominado por esfuerzos de tracción aunque su valor sea muy pequeño $(2,88 \mathrm{kPa})$ en comparación con la magnitud de las compresiones.

\subsubsection{Análisis del comportamiento estructural del material de relleno}

El análisis efectuado a partir de la Figura 8 permite concluir que el material de relleno presenta grandes extensiones superficiales de esfuerzos a la tracción cercanos a los 3,4 kPa, 
lo cual representa un $9,23 \%$ del valor de las compresiones que en su interior se producen cuyo máximo valor corresponde a $36,8 \mathrm{kPa}$. El sentido en que se distribuyen los esfuerzos en la gráfica de tonos de derecha a izquierda es análogo al que tiene el puente de arriba hacia abajo, lo que permite asumir que las tracciones se presentan en la parte superior del puente y a medida que se desciende en la estructura este esfuerzo se convierte en compresión y aumenta progresivamente hasta llegar a su mayor valor.

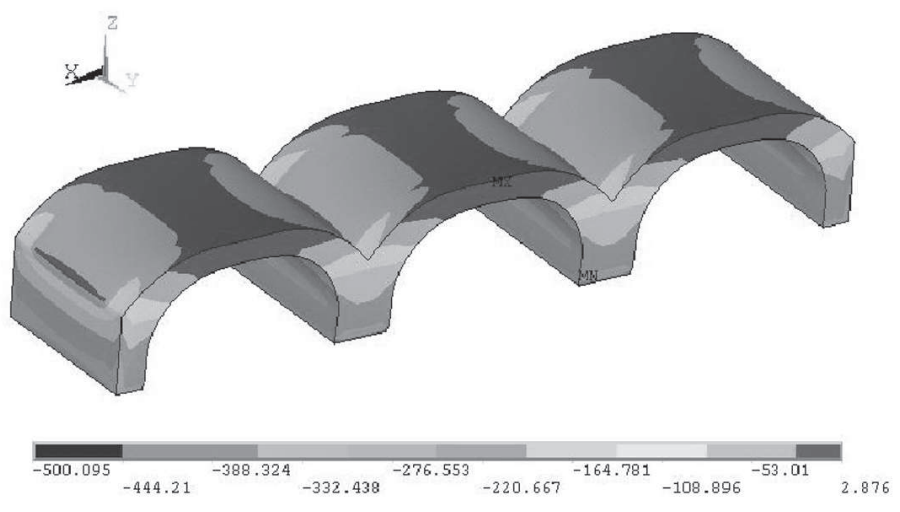

Figura 7. Distribución de esfuerzos en el eje Z en la zona de las bóvedas del puente La Libertad.
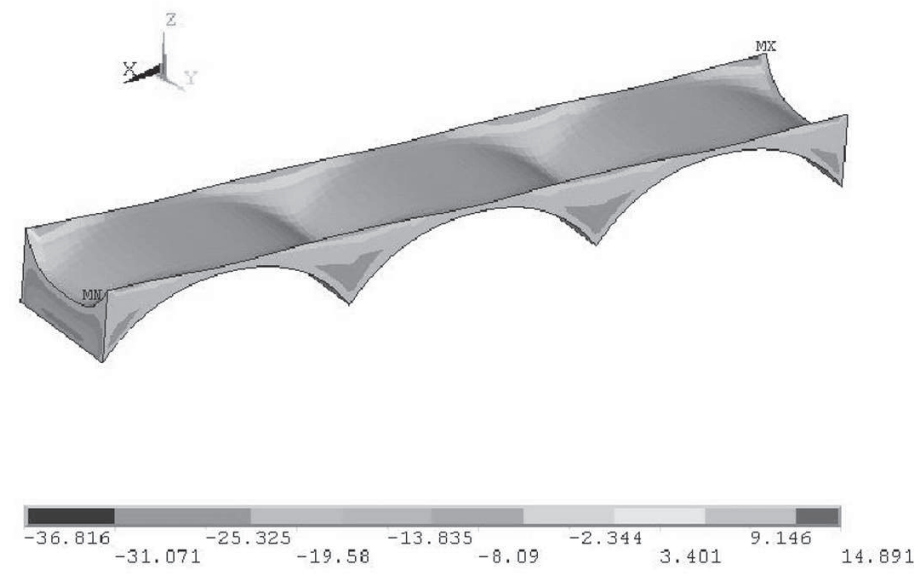

Figura 8. Esfuerzos en el eje Z del material de relleno del puente La Libertad.

De lo anterior se confirma que las mayores compresiones en el sentido vertical se presentan en los senos del puente. como se lee en la Figura 9. A medida que se desciende en altura, esta compresión se hace mayor, los esfuerzos se concentran en los senos y su máximo valor alcanza los $14,82 \mathrm{kPa}$ que representan aproximadamente mitades $50 \%$ del máximo valor en el sentido vertical o eje $Z$ cuyo valor es $37 \mathrm{kPa}$. 


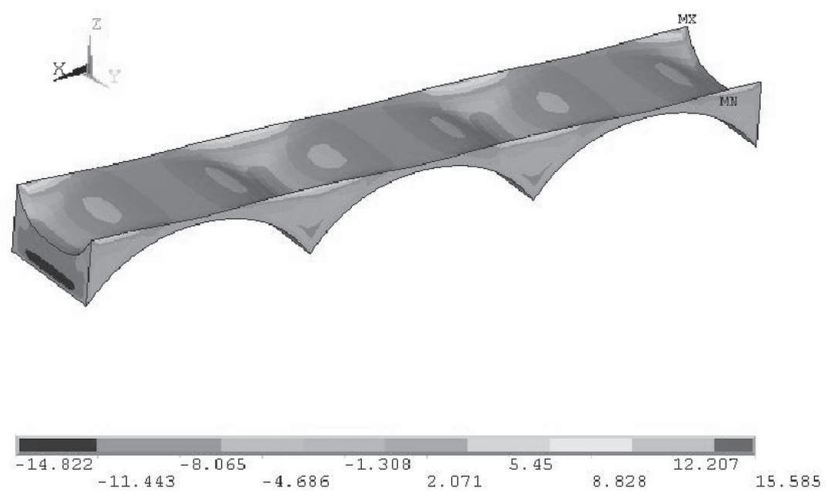

Figura 9. Esfuerzos en el eje $\mathrm{X}$ del material de relleno del puente La Libertad.

El análisis de la Figura 10 permite concluir que los esfuerzos para el material de relleno tienen un comportamiento similar en el sentido transversal o eje $Y$ al que presenta a través del sentido longitudinal o eje $\mathrm{X}$, anteriormente descrito. Las mayores compresiones también están ubicadas en los senos del puente y su valor de $15,95 \mathrm{kPa}$, lo que es también aproximadamente la mitad del máximo valor en el sentido vertical; mientras que las mayores tracciones aumentan en un $100 \%$ para este sentido. Sin embargo, dichas tensiones se presentan en el mismo sitio.

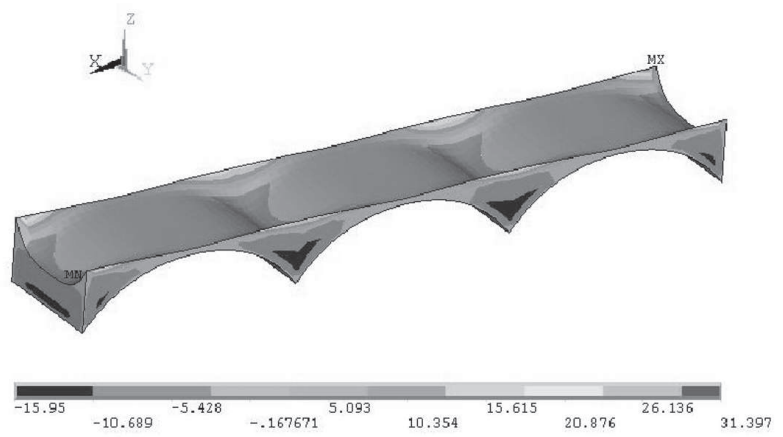

Figura 10. Esfuerzos en el eje $Y$ del material de relleno del puente La Libertad.

El desplazamiento del material de relleno es el que presenta el mayor valor de toda la estructura ya que el módulo de elasticidad $(E)$ obtenido en pruebas de laboratorio para este material es muy bajo (8500 kPa). Su valor es de $4,40 \mathrm{~mm}$ y se aprecia en la Figura 11 en lo profundo de los senos del puente. Complementando esta observación con las descritas en las figuras anteriores se puede concluir que en los senos se manifiesta la mayor vulnerabilidad del puente frente a cargas diferentes a su peso propio, zonas donde el material de relleno presenta sus mayores esfuerzos a la compresión así como los máximos desplazamientos. Este modelo expresa la realidad ya que en el pavimento del puente La Libertad los mayores agrietamientos se dan precisamente sobre estas zonas. 


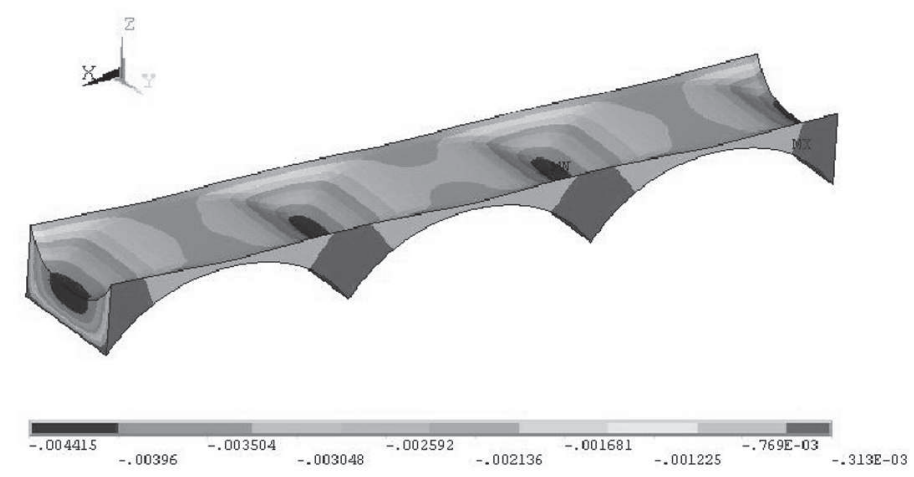

Figura 11. Desplazamientos en el eje $Z$ del material de relleno del puente La Libertad.

\subsubsection{Análisis del comportamiento global del puente}

El modelo que muestra la Figura 12 es analizado bajo fuerzas de gravedad debidas al peso propio de la estructura. Su lectura permite concluir que el puente está sometido mayoritariamente a esfuerzos de compresión que oscilan entre $42 \mathrm{kPa}$ y $442,8 \mathrm{kPa}$. Los mayores esfuerzos a compresión se concentran en las bases de las pilas centrales y tienen un valor aproximado de $500 \mathrm{kPa}$. El puente en su conjunto presenta extensas franjas sometidas a esfuerzos de tracción las cuales se dan en la parte alta de la estructura, concentrados principalmente en el material de relleno y los tímpanos. Sin embargo se debe advertir que estas tensiones tienen una baja magnitud comparada con la de los esfuerzos de compresión, llegando solamente al 3,1\% de su valor.

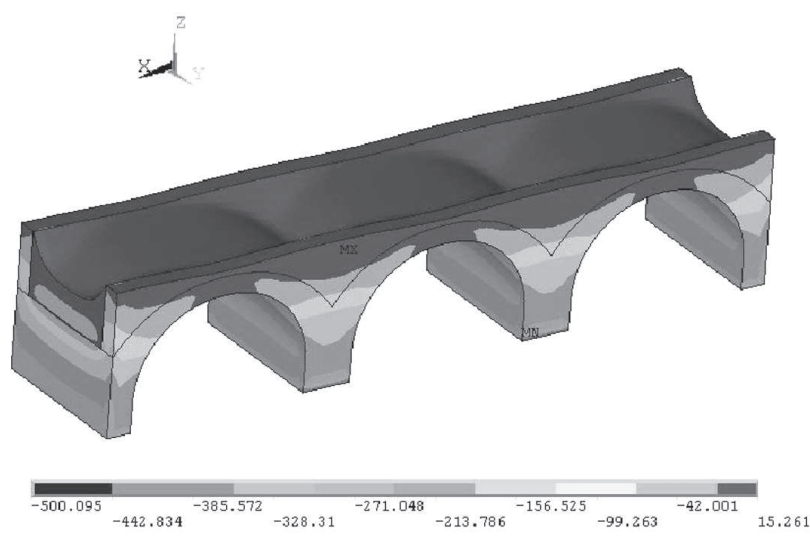

Figura 12. Distribución de esfuerzos en el eje Z en la obra de mampostería del puente La Libertad.

En el sentido longitudinal o eje X, los mayores esfuerzos de tracción equivalen a 165,3 kPa y son prácticamente iguales a los mayores esfuerzos de compresión los cuales se aprecian en la Figura 13 que son equivalentes a 172,16 kPa, ambos situados en los tímpanos del 
puente. Vale la pena aclarar que es precisamente en los tímpanos donde se presentan las únicas fracciones sometidas a tracción si nos referimos al material mampuesto. En las roscas predomina la compresión y su mayor valor allí es cercano a los 134,7 kPa lo cual se da aproximadamente en un cuarto de la luz de cada rosca. Por su parte el material de relleno es dominado por esfuerzos de compresión así como las roscas pero con una magnitud menor, cercana a los $15 \mathrm{kPa}$.

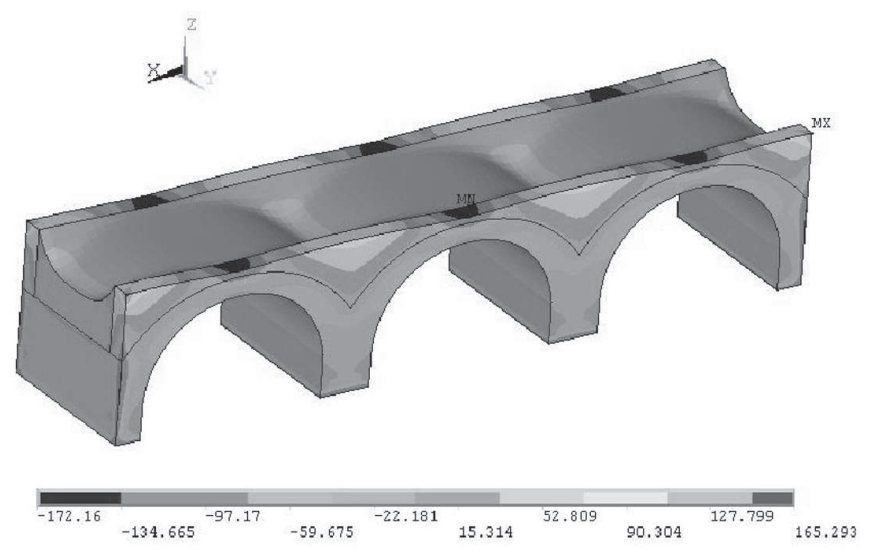

Figura 13. Distribución de esfuerzos en el eje X en la obra de albañilería del puente La Libertad.

Prácticamente la estructura en su conjunto está sometida a esfuerzos de tracción en el sentido transversal de la misma o eje $\mathrm{Y}$ con valores inferiores a $47 \mathrm{kPa}$, valores muy bajos comparados con las tensiones presentadas en los otros dos sentidos. El cuadro de tonos de la Figura 14 que enseña los resultados, expresa que existen esfuerzos de compresión que oscilan entre $19,3 \mathrm{kPa}$ y $153 \mathrm{kPa}$, sin embargo el puente muestra que las franjas que corresponden a estos valores ocupan una región muy pequeña si se toma como referencia el puente en su totalidad, aunque son de mayor magnitud en comparación con los esfuerzos de tracción, quienes son apenas un $31 \%$ de los de compresión.

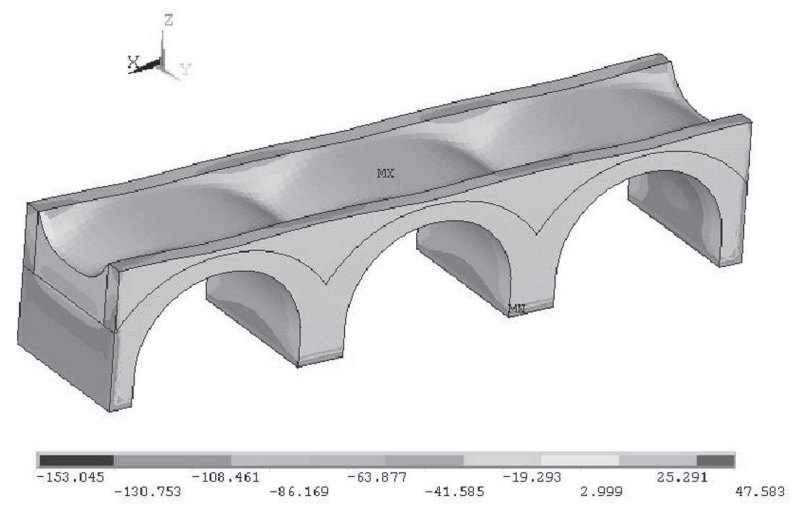

Figura 14. Distribución de esfuerzos en el eje Y en la obra de albañilería del puente La Libertad. 
Como era de esperarse, los mayores desplazamientos se dan en el sentido vertical o eje $Z$ de la estructura y están representados en la Figura 15. Los mayores valores de desplazamiento se dan en el material de relleno los cuales se muestran en los senos del puente cercanos a 4,4 mm. El máximo desplazamiento en las roscas se presenta en el centro del puente el cual es de $1,3 \mathrm{~mm}$.

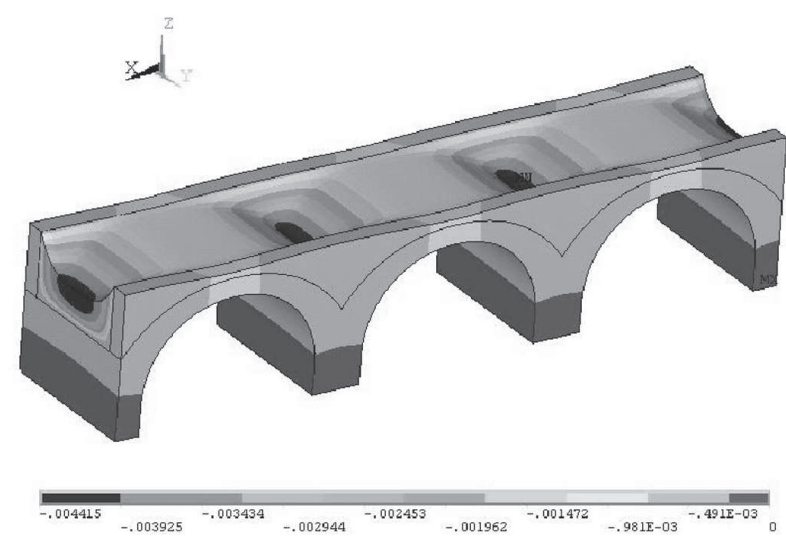

Figura 15. Desplazamientos en el eje Z en la obra de albañilería del puente La Libertad.

\section{CONCLUSIONES}

Si bien el puente pudo haberse reducido en una cuarta parte para ser modelado en el programa informático en virtud de ser una estructura simétrica, su escala permitió un análisis integral lo cual es mejor a efectos prácticos puesto que lo primero implicaría nuevas condiciones de borde, en tanto que la visualización del modelo en su contexto global permite una mejor lectura de los resultados. Así es entonces posible afirmar que la distribución de esfuerzos de compresión cuenta con su mayor valor sobre todas las bóvedas en el primer cuarto de cada una de sus luces y que el mayor esfuerzo de tracción de la estructura se presenta en el sentido longitudinal con un valor correspondiente a 165,3 kPa. En los sentidos transversal y vertical las tracciones son mucho más bajas pero se extienden en mayor proporción.

Los análisis permitieron establecer que los esfuerzos máximos a compresión que actúan en el puente son cercanos a los $500 \mathrm{kPa}$ y se presentan en la mampostería de la rosca; los esfuerzos máximos de tracción rondan los $165 \mathrm{kPa}$ y se sitúan en la zona de los tímpanos. Si comparamos estos resultados con los esfuerzos admisibles estimados para la mampostería (590kPa para compresión axial y 980 kPa para compresión por flexión) además de los esfuerzos admisibles para tracción por flexión en mampostería no reforzada contemplados en la norma NSR-98 (190 kPa y $370 \mathrm{kPa}$ conforme a la Tabla D-1-2- contenida en [2]), se puede concluir que la estructura se encuentra todavía dentro de un rango de seguridad frente a esfuerzos y con una tolerancia aun de $90 \mathrm{kPa}$ en cuanto a esfuerzos admisibles por compresión axial y $25 \mathrm{kPa}$ en cuanto a esfuerzos admisibles para tracción por flexión. 
Se sabe también que la resistencia a la compresión obtenida mediante ensayos fue para el ladrillo de $7350 \mathrm{kPa}$ y para el mortero de $350 \mathrm{kPa}$, dando en su conjunto como resultado una resistencia a la compresión de la mampostería igual a $2970 \mathrm{kPa}$, por tanto los esfuerzos actuantes solamente alcanzan el $16.8 \%$ de los esfuerzos resistentes, con lo cual se ratifica la premisa de la teoría de estado límite según la cual la resistencia a la compresión de los materiales se puede considerar infinita, es decir, se presentan cargas muy bajas en comparación con la resistencia última del material.

En el conjunto de la estructura los esfuerzos son relativamente bajos, y consecuentemente con esto, el mayor desplazamiento del material mampuesto es pequeño $(1,3 \mathrm{~mm})$ ante un esfuerzo de $500 \mathrm{kPa}$. Por su parte, el mayor desplazamiento ocurre en el material de relleno $(4,4 \mathrm{~mm})$ bajo un esfuerzo de solo $37 \mathrm{kPa}$ puesto que su módulo de elasticidad es también muy bajo (8500 kPa).

Los resultados obtenidos son consecuentes con las valoraciones obtenidas a partir de la inspección visual de la estructura. No hay señales de fisuras causadas por fallo en la capacidad resistente en la mampostería, así como no hay evidencias de desplomes en los tímpanos o cualquier otra situación que pudiese amenazar con el colapso de la estructura, lo que resta valor a propuestas de intervención orientadas a reforzar la obra de albañilería mediante pórticos de confinamiento o arcos de hormigón bajo las bóvedas; sin embargo, el material de relleno es muy compresible y en condiciones de saturación de humedad puede tener efectos contraproducentes hacia la estabilidad del conjunto, en especial por el efecto de empuje horizontal sobre los tímpanos laterales. En este sentido, son saludables para el puente La Libertad acciones orientadas a mejorar las condiciones del relleno o reemplazarlo por uno de mejor calidad; además deberán mejorarse las condiciones de evacuación de las aguas que se filtran hacia el interior de los senos a través del pavimento.

El fenómeno de socavación que presentan las pilas centrales apoyadas en el lecho del río, puede -a mediano plazo- casar una transformación en las condiciones de equilibrio de la estructura. Estudios posteriores en torno al puente de La Libertad que contemplen condiciones dinámicas a causa del tráfico vehicular y cargas sísmicas deben ser desarrollados.

\section{AGRADECIMIENTOS}

Los autores desean agradecer el respaldo dado por la Dirección de Investigaciones de la Universidad Nacional de Colombia, sede Manizales, a las dos investigaciones que soportan este artículo: Puentes de bóvedas en la región del Alto Cauca. Fase l: en busca de una tradición constructiva olvidada (2005-2006), y Fase II: modelización numérica y monitoreo de salud estructural (en curso). Igualmente, destacan la colaboración de la Arquitecta Constructora Marisol Caicedo Muñoz quien coordinó los análisis de laboratorio y al Ingeniero Civil John Fredy Osorio, quien operó los programas informáticos mencionados en este artículo. 


\section{REFERENCIAS BIBLIOGRÁFICAS}

[1] ANTOINE, A; (1994). Derivation of the in-plane elastic characteristics of masonry through homogenization theory; En: Int. J. Solids Structures, Vol. 32, pp. 137-163.

[2] Asociación Colombiana de Ingeniería Sísmica (2002). NSR-98. Normas colombianas de diseño y construcción sismorresistente. Bogotá: ACIS.

[3] BUHAN, P. \& FELICE, G.; (1997). A homogenization approach to the ultimate strength of brick masonry; En: J. Mech. Phys. Solids, Vol. 45; pp. 1085-1104.

[4] CARBONE, V.I. \& CODEGONE, M.; (2005). Homogenization Process of Stratified Masonry; En: Mathematical and Computer Modelling, Vol. 42; pp. 375-380.

[5] CAVICCHI, A. \& GAMBAROTTA, L.; (2005). Collapse analysis of masonry bridges taking into account arch-fill interaction; En: Enginnering Structures, Vol. 27; pp. 605615.

[6] DAS, B.M., (2006). Principios de ingeniería de cimentaciones. México. Thompson Intern.

[7] FANNING P.J., BOOTHBY T.E., Roberts B.J.; (2001). Three-dimensional modeling and full-scale testing of stone arch bridges; En: Computers and Structures, Vol. 79; pp. 2645-2662.

[8] Instituto Colombiano de Normas Técnicas y Certificación (1999). Ensayo para determinar la granulometría por tamizado. Bogotá. ICONTEC.

[9] Instituto Colombiano de Normas Técnicas y Certificación (2000). Método de ensayo para determinar la resistencia a la compresión en frío y el módulo de ruptura de refractarios. Bogotá. ICONTEC.

[10] Instituto Colombiano de Normas Técnicas y Certificación (2004). Determinación de la resistencia de morteros de cemento hidráulico usando cubos de $50 \mathrm{~mm}$ ó $50,8 \mathrm{~mm}$ de lado. Bogotá. ICONTEC.

[11] MOLANO, M.A. (2005): Rehabilitación sísmica de mampostería no estructural mediante listones de madera. Página consultada el 20 de agosto de 2007. En:

http://www.javeriana.edu.co/biblos/tesis/ingenieria/tesis125.pdf ; p.38.

[12] MUÑOZ, E. \& VALBUENA, E.; (2004). Evaluación del estado de los puentes de acero de la red vial de Colombia; En: Boletín técnico IMNE, Vol. 3; pp. 1-16.

[13] ROYLES, D. \& HENRY, A.W.; (1991). Models tests of masonry arches; En: Proc. Inst. Civ. Engrs Part 2, Vol. 91; pp. 299-321. 